\title{
With thanks to our 2015 peer reviewers
}

We are grateful to the following people for their significant contribution to Health Promotion and Chronic Disease Prevention in Canada as peer reviewers in 2015. Their expertise ensures the quality of our journal and promotes the sharing of new knowledge among peers in Canada and internationally.

José Almirall

Shazhan Amed

Tracie Barnett

Lana Beasley

Julie Bernier

Beatrice Boucher

Aimée Campeau

Yue Chen

Kristin Connor

Sarah Connor Gorber

Chris de Gara

Jessica Di Giovanni

Maureen Dobbins

Frank Elgar

Tara Flemington

Sarah Flicker

Robert Gates

Danijela Gasevic

Jenny Gray

Martine Hébert

Kathleen Hegadoren

Margaret Herbert

Shelly L. Jackson

Ian Janssen

Teresa Janz
Janet Elizabeth Jull

Lisa Kakinami

Glenn Keays

Colin King

Richard Krugman

Eric Lavigne

Allana LeBlanc

Isra Levy

Karen Liller

Larisa Lotoski

Wei Luo

Patti-Jean Naylor

Candace Nykiforuk

Alison Macpherson

Steven McFaull

Nazeem Muhajarine

Gillian Mulvale

Martin Noël

Lawrence Paszat

Scott B. Patten

Louise Pelletier

Cheryl Peters

Jennifer Petkovic

William Pickett

Robert Platt
Keith Power

Robert Prosser

Kirstein Rummery

Kelly Russell

Tara Sampalli

Travis Saunders

Jerry A. Schultz

Kevin Schwartz

Mark Smith

Richard Stanwick

Michael Stevenson

Larry Svenson

Wendy Thompson

Ellen Toth

Paul Veugelers

Charlotte Waddell

Christine Walsh

Darren Warburton

Sharon Warren

Christine White

Helene White

Andreas T. Wielgosz

Russell Wilkins 